\title{
Efficacy of the Pilates method for pain and disability in patients with chronic nonspecific low back pain: a systematic review with meta-analysis
}

\begin{abstract}
Gisela C. Miyamoto ${ }^{1}$, Leonardo O. P. Costa ${ }^{1,2}$, Cristina M. N. Cabral ${ }^{1}$
ABSTRACT | Objective: To systematically review the available evidence on the efficacy of the Pilates method in patients with chronic nonspecific low back pain. Method: Searches were performed in MEDLINE, EMBASE, PEDro, SciELO, LILACS, CINAHL and CENTRAL in March 2013. Randomized controlled trials that tested the effectiveness of the Pilates method (against a nontreatment group, minimal intervention or other types of interventions) in adults with chronic low back pain were included regardless the language of publication. The outcome data were extracted from the eligible studies and were combined using a meta-analysis approach. Results: The searches identified a total of 1,545 articles. From these, eight trials were considered eligible, and seven trials were combined in the meta-analysis. The comparison groups were as follows: Pilates versus other types of exercises ( $n=2$ trials), and Pilates versus no treatment group or minimal intervention ( $\mathrm{n}=4$ trials) for short term pain; Pilates versus minimal intervention for short-term disability ( $\mathrm{n}=4)$. We determined that Pilates was not better than other types of exercises for reducing pain intensity. However, Pilates was better than a minimal intervention for reducing short-term pain and disability (pain: pooled mean difference $=1.6$ points; $95 \%$ CI 1.4 to 1.8; disability: pooled mean difference $=5.2$ points; $95 \%$ CI 4.3 to 6.1 ). Conclusions: Pilates was better than a minimal intervention for reducing pain and disability in patients with chronic low back pain. Pilates was not better than other types of exercise for short-term pain reduction.
\end{abstract}

Keywords: backache; exercise therapy; rehabilitation.

HOW TO CITE THIS ARTICLE

Miyamoto GC, Costa LOP, Cabral CMN. Efficacy of the Pilates method for pain and disability in patients with chronic nonspecific low back pain: a systematic review with meta-analysis. Braz J Phys Ther. 2013 Nov-Dec; 17(6):517-532. http://dx.doi.org/10.1590/ S1413-35552012005000127

\section{Introduction}

Low back pain is a major health and socioeconomic problem and one of the main causes of disability and absenteeism ${ }^{1-4}$. It is estimated that between 11 and $84 \%$ of adults will experience an episode of low back pain at least once in their lives ${ }^{5}$, and approximately $40 \%$ of these patients will develop chronic low back pain lasting more than three months $s^{4,6,7}$. A systematic review of the prognosis of patients with low back pain has shown that both patients with acute low back pain and those with persistent low back pain improved within the first six weeks. However, after this period, the improvement slowed down, and low to moderate levels of pain and disability were present at one year, especially in patients with persistent pain ${ }^{8}$. Estimates show that low back pain leads to an expenditure of nine billion Australian dollars in Australia and 12 billion British pounds in the United Kingdom when direct and indirect costs are taken into account ${ }^{9}$.

The effect of the treatments recommended by guidelines for chronic low back pain is moderate ${ }^{4,10}$. Exercise is the physical therapy treatment for chronic low back pain that shows the most lasting and positive effects ${ }^{10}$. Pilates method exercises involve contractions of the deep abdominal muscles. When used in physical therapy practice, the method requires modifications, such as adaptations and simplifications of the traditional method. Such exercises are thus described as Pilates method-based exercises ${ }^{11}$.

For some time, Pilates method-based exercises were considered to have unknown value in the

\footnotetext{
${ }^{1}$ Master and Doctoral Program in Physical Therapy, Universidade Cidade de São Paulo (UNICID), São Paulo, SP, Brazil 
treatment of low back pain ${ }^{10}$. However, randomized controlled studies on this method began to be published in $2006^{12-14}$. Two systematic reviews without meta-analysis have been published on the use of the Pilates method to treat patients with chronic low back pain ${ }^{3,15}$, and the results showed improvement in pain and disability. In 2011, two systematic reviews with meta-analyses were published regarding Pilates method-based exercises ${ }^{16,17}$. The results of the first review ${ }^{16}$ showed that Pilates method-based exercises are superior to a minimal intervention for reducing chronic nonspecific low back pain after intervention. Conversely, the second ${ }^{17}$ review showed no significant improvement in pain and disability when groups that participated in Pilates methodbased exercises were compared with a control group or group performing lumbar stabilization exercises.

Although four systematic reviews have been published recently, the authors included studies that had not yet been published as journal articles indexed in databases that go through rigorous peer review (especially master dissertations or doctoral theses $)^{16,17}$. They also included studies of patients with discopathies ${ }^{15}$, which can directly interfere with the results of systematic reviews. The objective of this study was therefore to systematically review randomized controlled trials comparing the effectiveness of the Pilates method with a control group, other types of intervention or other types of exercises in terms of pain and disability in patients with chronic nonspecific low back pain.

\section{Method}

\section{Inclusion criteria}

The inclusion criteria for the analysis were studies that were randomized controlled trials of adults with chronic nonspecific low back pain that evaluated pain and/or disability; studies in which the primary treatment was based on Pilates method exercises compared with no treatment, minimal intervention, other types of intervention or other types of exercises; studies published in scientific journals between 1980 and 2013 and without restriction on language of publication.

\section{Search strategy}

Searches were conducted in the MEDLINE, EMBASE, PEDro, SciELO [Scientific Electronic Library Online], LILACS [Literatura Latino
Americana em Ciências da Saúde (Latin American and Caribbean Health Sciences Literature)], CINAHL [Cumulative Index to Nursing and Allied Health Literature] and CENTRAL [Cochrane Central Register of Controlled Trials] databases. The terms used in the searches were based on the search strategies of the Cochrane Back Review Group (Appendix 1). The last day of the search for articles was March 10, 2013. The searches were adjusted for each database because of the differences between the search engines of each of those databases. These adjustments followed the recommendations of the Cochrane Collaboration ${ }^{18}$.

\section{Study selection}

Two independent reviewers performed the first analysis based on the information provided by the title, abstract and keywords. In cases of disagreement between the reviewers, a third reviewer was consulted to achieve consensus.

\section{Evaluation of the methodological quality of the studies}

The methodological quality and statistical description of the studies were measured using the PEDro quality scale ${ }^{19-21}$ and these data were extracted from the PEDro database (www.pedro.org.au). The PEDro scale consists of the following items: 1) Were the eligibility criteria specified?,2) Were the subjects randomly allocated to groups?, 3) Was the allocation of the subjects concealed?, 4) Were the groups similar at baseline with regard to the most important prognostic indicators?, 5) Was there blinding of all the subjects who participated in the study?, 6) Did all therapists who administered the therapy do so blindly?, 7) Was there blinding of all assessors who measured at least one key outcome?, 8) Were measurements of at least one key outcome obtained for more than $85 \%$ of subjects initially allocated to the groups?, 9) Did all subjects for whom outcomes were measured receive the treatment or control condition as allocated or, when this was not the case, was an "intention to treat" data analysis performed for at least one of the key outcomes?, 10) Were the results of between-group statistical comparisons reported for at least one key outcome?, and 11) Did the study have both point measures and measures of variability for at least one key outcome? ${ }^{22}$.

PEDro quality scale scoring is between 0 and 10 points; the first item, relating to external validity, is not used to calculate the PEDro score. The reliability of the items on this scale ranged from good to 
excellent $^{22,23}$. Studies that were not available on the PEDro database were evaluated by two independent reviewers. In cases of disagreement between the reviewers, a third reviewer was consulted to achieve consensus.

\section{Data extraction}

To analyze the studies, data were extracted regarding participants' characteristics (age and duration of symptoms), intervention, duration and frequency of treatment sessions based on the Pilates method, the control group (no treatment, minimal intervention, other types of intervention or other types of exercises) and pain and disability outcomes at all assessment time-points. Meta-analysis was not performed when there was only one study for comparison or when statistical heterogeneity was identified.

\section{Statistical analysis of the meta-analysis}

For the meta-analysis, data extraction was performed based on the data presented in the included articles, with data extracted from the text, tables or figures and expressed as mean and standard deviation (Corel Draw software Version 3 was used to extract data from figures). This procedure was performed for the outcomes short-term pain and disability; the Pilates method was compared with no treatment, minimal intervention, other types of intervention or other types of exercises. Sensitivity analyses were performed to identify studies with high statistical heterogeneity and to determine whether the methodological quality of the eligible articles and the number of sessions conducted influenced the size of the observed effects. The study was removed from the meta-analysis when the presence of statistical heterogeneity and/or an effect of methodological quality or number of sessions were detected. An analysis of publication bias was also performed via the visual inspection of funnel plots ${ }^{18}$. Studies with larger samples had a higher weight in the metaanalysis results ${ }^{24}$. Means and standard deviations were converted into scales from 0 to 10 points for the pain outcome and 0-100 points for disability, standardizing the scales that other studies used to perform the meta-analysis. $I^{2}$ was calculated using Comprehensive Meta Analysis software Version 2 for a statistical analysis of heterogeneity, which describes the percentage of variation in the estimates of effects caused by heterogeneity rather than sampling error. A value above $50 \%$ can be considered substantial heterogeneity. When the values were statistically homogeneous, the average effects (difference between the weighted averages) were calculated using a fixed-effect model $\left(\mathrm{I}^{2}<50 \%\right)$. When the values were statistically heterogeneous, the estimates of average effects (difference between the weighted averages) were obtained using a random-effects model $\left(\mathrm{I}^{2}>50 \%\right)$. The standardized mean difference with a confidence interval of $95 \%$ was used. Forest plots, funnel plots and meta-analyses were also calculated using the Comprehensive Meta Analysis software.

\section{Results}

\section{Study selection and characteristics}

A total of 1,545 articles were found, of which eight studies $^{13,14,25-30}$ were considered eligible. Of these, seven $^{13,14,26-30}$ were included in the meta-analysis (Figure 1). Two studies were excluded from the meta-analysis: one study ${ }^{25}$ evaluated three different regimens of the Pilates method and therefore had no comparison group for analysis, and another study ${ }^{13}$ was excluded from the meta-analysis for the pain outcome based on the sensitivity analysis because its inclusion considerably increased the level of heterogeneity of the meta-analysis. In one study ${ }^{26}$ comparing the vertical ground-reaction force between the control group (healthy subjects) and the group with low back pain (divided into two groups: Pilates group and no treatment group), only the groups of patients with low back pain were considered. Table 1 shows the characteristics of the studies in descriptive form, and Table 2 shows the details of the studies.

\section{Methodological quality results}

In evaluating the methodological quality using the PEDro scale, the scores for the seven articles were already available on the PEDro database ${ }^{13,14,25,26,28-30}$. One article $^{27}$ was evaluated by two independent reviewers because it was still not available. The scores ranged from 2 to 8 points on a scale of 0 to 10 points (Table 3). All of the studies lost points on items related to the blinding of the patient and therapist, and only four studies ${ }^{13,14,27,30}$ blinded the assessor. A score of 2 points was observed in only one study ${ }^{25}$, and a score of 8 points, the maximum considered for this type of study as it is not possible to blind therapist and patients, was observed in two studies ${ }^{14,30}$.

\section{Treatment effects}


The visual inspection of the funnel plot did not identify publication bias for any of the three metaanalyses. The sensitivity analyses considering the methodological quality and the number of sessions conducted did not significantly influence the results of the meta-analyses. Therefore, no study was removed from the meta-analyses. It was not possible to analyze the influence of the type of exercises on the metaanalyses because the descriptions of the exercises performed in eligible studies were very brief.

\section{Comparison of the Pilates method with minimal intervention for pain outcome}

Four studies ${ }^{14,26,27,30}$ evaluated pain before and after intervention $(n=171)$. The study by Rydeard et al. ${ }^{14}$ compared the Pilates method with primary care, such as medical appointments when needed $(n=39)$. Pilates method-based floor exercises were performed initially as static exercises; they progressed to exercises involving hip extension movements and then to exercises on the Reformer, with 12 one-hour sessions conducted in addition to a home-based program of 15 minutes, six days a week for four weeks. Da Fonseca et al.'s study ${ }^{26}$ compared the Pilates method with no exercise $(n=17)$. In that study, floor exercises were carried out based on the Pilates method. These exercises involved limb movement with a static back position progressing to a dynamic back position; onehour sessions were held twice a week for a total of 15 sessions. Quinn et al.'s study ${ }^{27}$ compared the Pilates method with no treatment $(n=29)$. In this study, Pilates exercises were performed based on O'Brien's

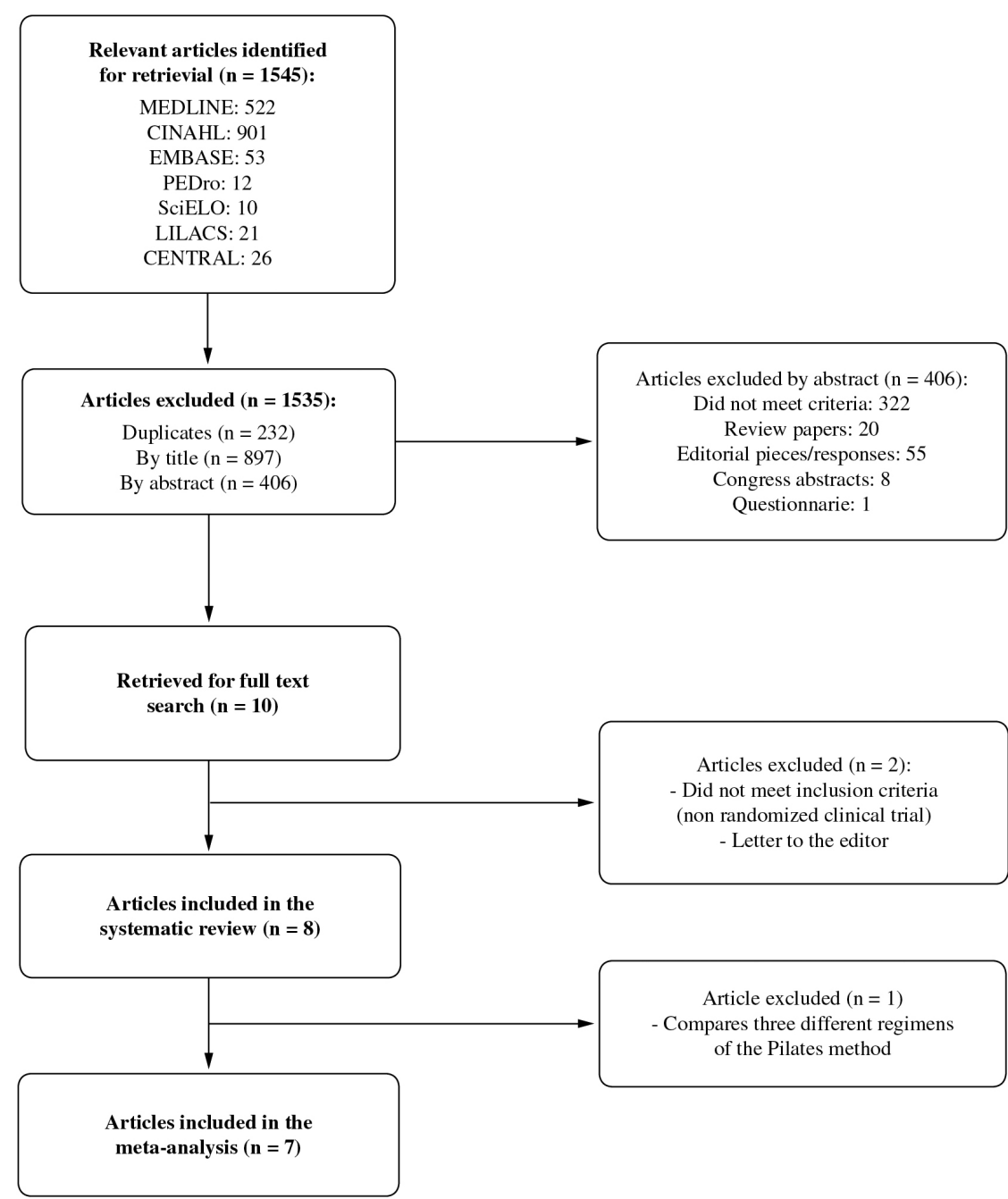

Figure 1. Selection process for studies included in the analysis. 
Table 1. Study characteristics.

\section{Authors}

Gladwell et al. ${ }^{13 * * * *}$

Rydeard et al. ${ }^{14 * * * * *}$

Rajpal et al. ${ }^{28 * *}$

Curnow et al. ${ }^{25}$

Da Fonseca et al. ${ }^{26 *}$

Quinn et al. ${ }^{27 * * * * *}$

\section{Participants}

Intervention

Mean age: CG: 45.9 years; PG: 36.9 years; CG: Normal activities and pain relief $\mathrm{n}=34$

PG: Pilates method-based floor exercises with Gender: CG: 4 male and 10 female; PG: 3 strengthening of abdominal, gluteus and back male and 17 female Duration of symptoms (mean $\pm \mathrm{SD})$ : CG: 139 \pm 148 ; PG: $115 \pm 101$ months muscles and progression with movement of the limbs, 6 sessions with 1 hour of duration for 6 weeks

Mean age: CG: 34 years; PG: 37 years; $\mathrm{n}=39$

CG: Normal care, such as medical appointments when necessary

Gender: CG: 8 male and 13 female; PG: 6 PG: Static Pilates method-based-exercises in male and 12 female

Duration of symptoms (median (interquartile range)): CG: 108 (12-240) months; PG: 66 (6-324) months with the following progression: addition of hip extension and exercises in the Reformer, 12 sessions with 1 hour of duration and a homebased program (duration of 15 minutes) for 4 weeks

Mean age: CG: 21.6 years; PG: 22.1 years; 30 consecutive sessions

$\mathrm{n}=32$

Gender: female

CG: McKenzie for sitting and standing posture correction, 3 repetitions performed 15-20 times

Duration of symptoms: $>3$ months in both per day groups

PG: Pilates method-based exercises on the floor, with breathing exercises and deep abdominal muscle contraction in supine, quadruped and sitting positions, 10 repetitions of 10 seconds

No specification of the participants

18 sessions for 6 weeks characteristics; $n=39$

The 3 groups performed 4 Pilates-based exercises on the floor; 2 exercises involved abdominal contraction, one involved lateral elevation of lower limbs in supine and one involved back muscles contraction, 40 repetitions of each exercise.

PG A: no additional exercises

PG B: exercises and relaxation, during 3-5 minutes

PG C: exercises, relaxation (for 3-5 minutes) and posture training involving hip flexion and eccentric psoas contraction (20 repetitions)

Mean age: CG: 34.4 years; PG: 31.6 years; CG: No intervention

$\mathrm{n}=17$

Gender: 5 male and 12 female

PG: Pilates method-based floor exercises (with the activation of deep abdominal muscles), Duration of symptoms: $>6$ months in both with the following progression: lower limb groups movements with static back position and dynamic back position, 15 sessions of 1 hour duration, 2 sessions per week

Mean age: CG: 44.1 years; PG: 41.8 years; CG: No intervention $\mathrm{n}=29$

PG: Pilates method-based exercises based on the Body Control Pilates program ${ }^{31}, 6$ to 8

Gender: female

1-hour sessions and 15 minutes at home, 5 times Duration of symptoms (mean $\pm \mathrm{SD})$ : CG: 49.2 (49.2) months; PG: 57.6 (38.4) months per week for 8 weeks

*CG: control group; PG: Pilates group. *Studies included in the comparison between Pilates and minimal intervention considering pain at short term. **Studies included in the comparison between Pilates and other types of exercises considering pain at short term. ***Studies included in the comparison between Pilates and minimal intervention considering disability at short term. 
Table 1. Continued...

\section{Authors}

Wajswelner et al. ${ }^{29 * *}$

Miyamoto et al. ${ }^{30 * * * * *}$

\section{Participants}

Intervention

Mean age: CG: 48.9 years; PG: 49.3 years; 12 sessions, 1-hour duration, for 6 weeks, with $\mathrm{n}=87$ exercises prescribed at home

Gender: CG: 20 male and 23 female; PG: CG: General exercises used in the treatment 19 male and 25 female Duration of symptoms (mean \pm SD): CG:14.2 \pm 12.7 ; GP: $13.6 \pm 14.2$ years (e.g., stationary bike, stretching, resistance training) and 4 exercises performed at home PG: 6 to 12 Pilates method-based exercises in the Reformer and Cadillac and 1 to 4 Pilates method-based exercises performed at home on the floor or with support of a chair or wall, involving back exercises in all planes of movement

Mean age: CG: 38,3 years; PG: 40,7 years; 12 sessions for 6 weeks, 2 times per week $\mathrm{n}=86$ GC: Educational booklet and 12 telephone calls

Gender: CG: 9 male and 34 female; PG: 7 during a 6-week period male and 36 female GP: Pilates method-based floor exercises Duration of symptoms (mean $\pm \mathrm{SD})$ : $\mathrm{CG}$ : 56.7 \pm 53.5 ; PG: $73.3 \pm 79.6$ months ing deep abclominal muscle contraction with stretching or strengthening of the back and lower limb muscles; 12 sessions, 1-hour duration, for 6 weeks

*CG: control group; PG: Pilates group. *Studies included in the comparison between Pilates and minimal intervention considering pain at shor term. $* *$ Studies included in the comparison between Pilates and other types of exercises considering pain at short term. ***Studies included in the comparison between Pilates and minimal intervention considering disability at short term.

Body Control Pilates program ${ }^{31}$, which consists of six to eight sessions lasting one hour and 15 minutes at home, five times a week at home for eight weeks. The study by Miyamoto et al..$^{30}$ compared Pilates method treatment with giving patients an educational booklet $(\mathrm{n}=86)$. The Pilates method-based floor exercises involved deep abdominal muscle activation exercises and exercises to strengthen and stretch the spinal and lower limb muscles, with 12 one-hour sessions over six weeks.

For the meta-analysis of this comparison, a fixed-effect model was used $\left(\mathrm{I}^{2}=0 \%, \chi^{2}=1.16\right.$, $\mathrm{df}=3, \mathrm{P}=0.76)$ in which a statistically significant difference was found between the groups when the Pilates method was compared with no treatment or minimal intervention for pain in the short term (difference between means $=1.6$ points, $95 \%$ CI 1.4 to 1.8 ; Figure $2 \mathrm{~A}$ ).

\section{Comparison of Pilates method with other types of exercises for the pain outcome}

For this analysis, two studies ${ }^{28,29}$ were included in the meta-analysis $(\mathrm{n}=119)$. Rajpal et al.'s study ${ }^{28}$ compared the Pilates method with the McKenzie method ( $n=32)$. In this study, Pilates method-based floor exercises were used and were associated with breathing exercises with deep abdominal muscle contraction in the supine, quadruped and sitting positions, with 10 repetitions of 10 seconds performed in each position. These were compared with McKenzie exercises, which involve sitting and standing posture correction, with three repetitions performed 15 to 20 times per day. Wajswelner et al.'s study $^{29}$ compared the Pilates method with general exercises (stationary bike, stretching and resistance training; $\mathrm{n}=87$ ), in which six to 12 supervised Pilates method-based exercises were performed on the Reformer and Cadillac, and one to four floor exercises were performed at home over 12 one-hour sessions for six weeks.

For the meta-analysis of this comparison, a fixed-effect model was used $\left(\mathrm{I}^{2}=0 \%, \chi^{2}=0.69, \mathrm{df}=1\right.$, $\mathrm{P}=0.41)$. At short term, no statistically significant difference was found between exercises for pain (difference between means $=0.1$ points, $95 \%$ CI -0.3 to 0.6 ; Figure 2B).

\section{Comparison of the Pilates method with minimal intervention for the disability outcome}

For disability, four studies that compared the Pilates method with minimal intervention ${ }^{13,14,27,30}$ using pre- and postintervention evaluations were included in the meta-analysis $(\mathrm{n}=188)$. The study by 


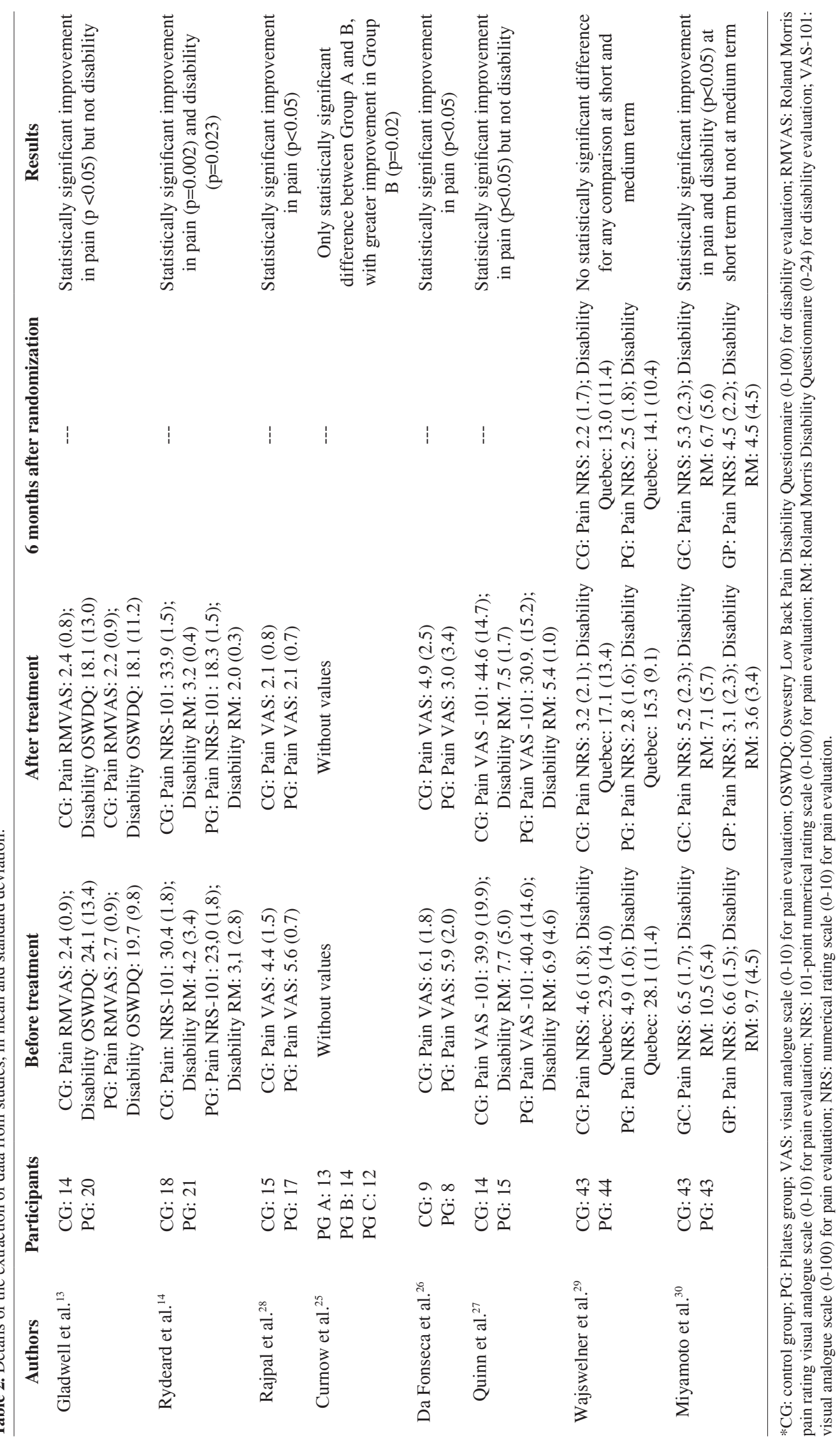


Miyamoto GC, Costa LOP, Cabral CMN

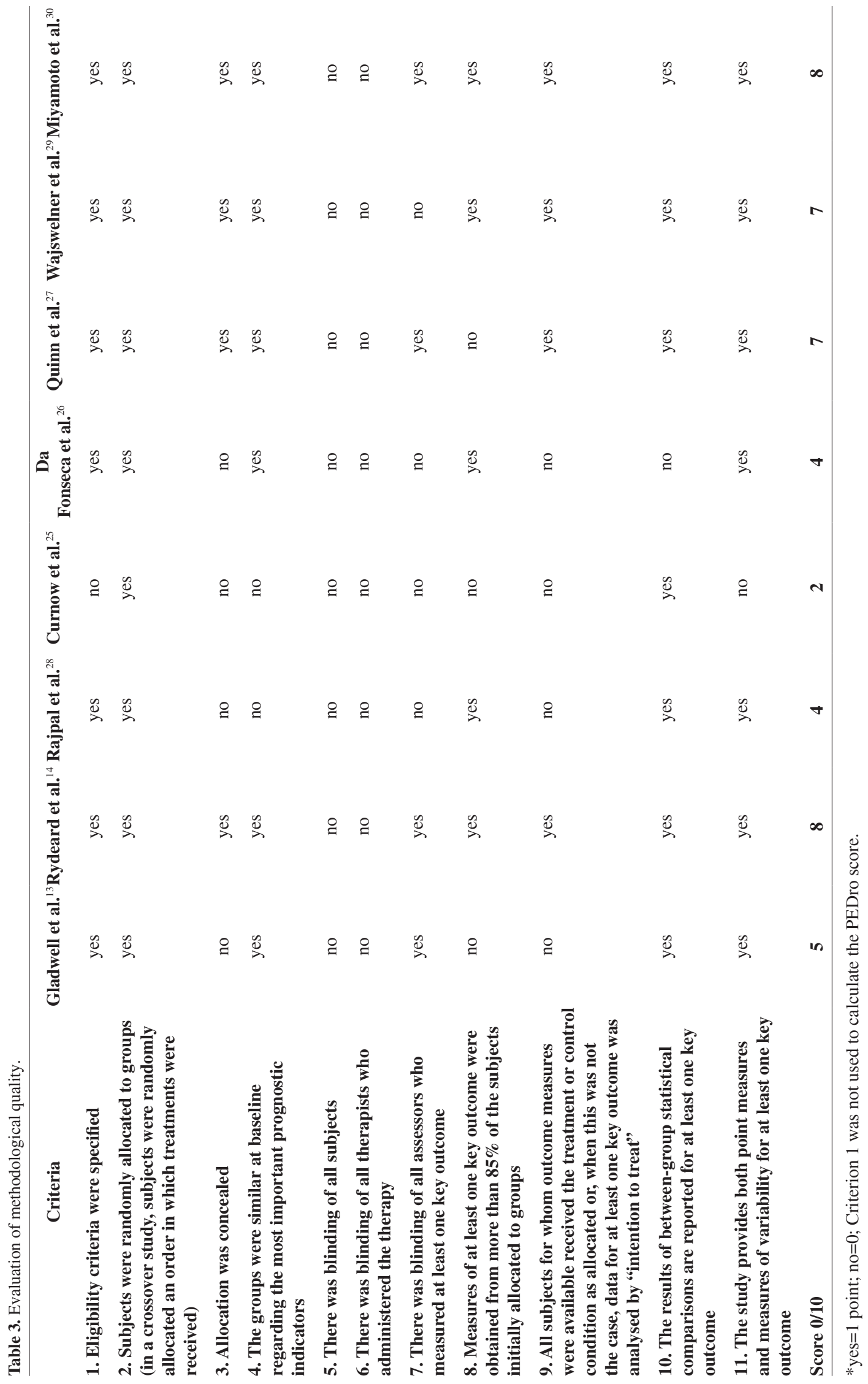

- 524 Braz J Phys Ther. 2013 Nov-Dec; 17(6):517-532 
Gladwell et al. ${ }^{13}$ compared the Pilates method with normal activities $(n=34)$. The exercises were basic Pilates method-based floor exercises that involved strengthening the abdominal muscles, gluteus muscles and spinal erectors and progressed to limb exercises, with a total of six sessions lasting one hour over six weeks. The other studies ${ }^{14,27,30}$ have been described above.

Figure $2 \mathrm{C}$ shows the results of this comparison for the disability outcome, using a random-effects model. A statistically significant improvement in disability favoring the Pilates method was observed at short term (difference between means $=5.2$ points, 95\% CI 4.3 to 6.1 ), with high level of heterogeneity $\left(\mathrm{I}^{2}=68 \%, \chi^{2}=9.35, \mathrm{df}=3, \mathrm{P}=0.02\right)$.

\section{Discussion}

This systematic review shows evidence that Pilates method-based exercises are more effective than no treatment or minimal intervention in the treatment of chronic nonspecific low back pain for the pain

A)

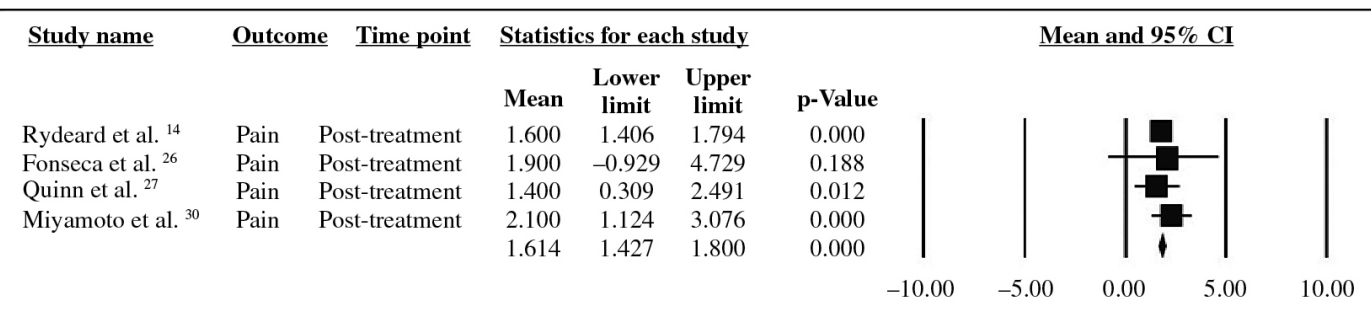

Favours Minimal Intervention Favours Pilates

B)

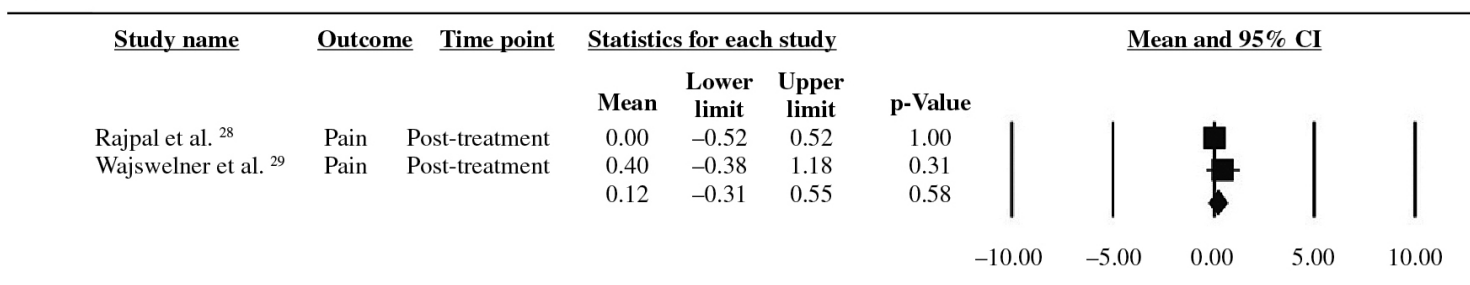

Favours Others Types of Exercises Favours Pilates

C)

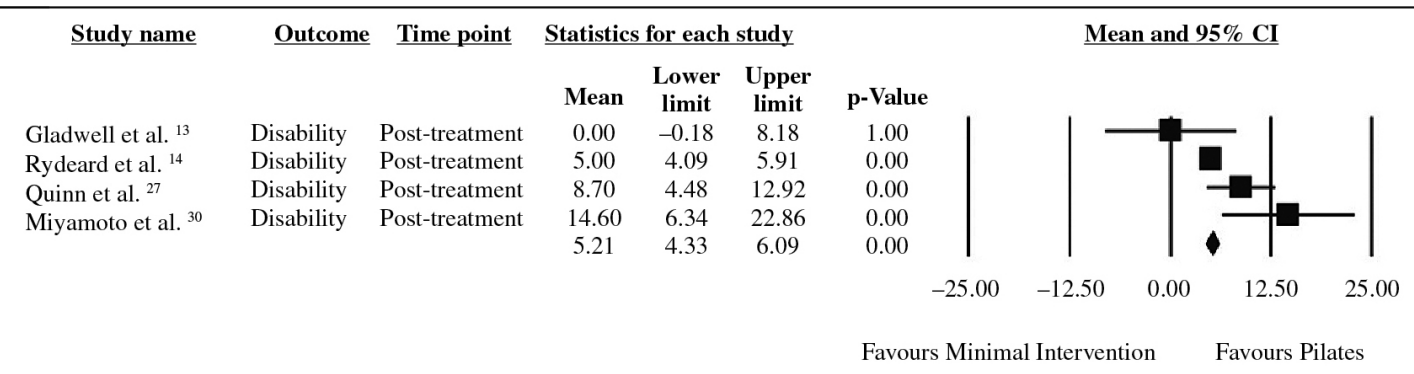

Figure 2. Forest plot of the results of the meta-analysis. A) Comparison of the Pilates method with minimal intervention for pain. The values shown are the average effects (difference between means) and the $95 \%$ confidence interval. The average effect was calculated using a fixed-effects model for short-term pain relief. B) Comparison of the Pilates method with other types of exercises for pain. The values shown are the average effects (difference between means) and the $95 \%$ confidence interval. The average effect was calculated using a fixed-effects model for short-term pain relief. C) Comparison of the Pilates method with minimal intervention for disability. The values shown are the average effects (difference between means) and the $95 \%$ confidence interval. The average effect was calculated using a random-effects model for short-term changes in disability. 
outcome. However, the difference between the means of this comparison was 1.6 points (on a scale of 0 to 10 points). This value does not represent a clinically significant improvement, as a minimum difference of 2 points is required ${ }^{32}$. The results show that Pilates method-based exercises and other types of exercises are equally effective for pain relief. These results are similar to other reviews of specific exercises ${ }^{10,33}$ that compare them with other types of exercises or other conservative interventions. For the disability outcome, greater improvement was observed for the Pilates method-based exercises compared with minimal intervention at short term. However, the difference between the means in this comparison was approximately 5 points (on a scale of 0 to 100 points), which does not represent a clinically significant improvement. A minimum difference of 10 points is required for there to be a clinical improvement ${ }^{32}$. There are few studies ${ }^{14,30}$ of high methodological quality with representative samples regarding the effectiveness of the Pilates method to treat chronic nonspecific low back pain; for this reason, the results should be interpreted with caution.

This systematic review only included studies published in full article form in journals indexed in databases that undergo rigorous peer review, which is usually performed by experts in the field. One study was excluded from the meta-analysis because it compared three different regimens of the Pilates method $^{25}$ and had no comparison group for analysis. Other systematic reviews with meta-analysis included three ${ }^{17}$ and four ${ }^{16}$ master dissertations and/or doctoral theses, studies that are difficult for the public to access and can present results without clinical relevance. Studies that have not been subjected to peer review may have a higher risk of bias or negative results and are not recommended for inclusion in systematic reviews ${ }^{34}$.

The present study's results are similar to those of another systematic review with meta-analysis ${ }^{16}$ on the same topic because the results show a significant difference in pain when the Pilates method is compared with minimal intervention and no statistically significant difference between groups for the same outcome when the Pilates method is compared with other types of exercises. However, the results do not corroborate the disability outcome, for which the present study shows a statistically significant difference in the short term. The difference between the results can be explained by the fact that the abovementioned systematic review ${ }^{16}$ included four studies that were not published in journals indexed in databases ${ }^{31,35-37}$. In addition, three studies that were included ${ }^{12,36,37}$ had low methodological quality (three to four points on a nine-point scale, the Delphi List), and the effect of the methodological quality of those studies on the meta-analyses was not assessed.

The results of the present study are in opposition to those obtained in another systematic review with meta-analysis ${ }^{17}$, as our results showed a significant difference for pain and disability when the Pilates method and minimal intervention were compared. However, the results corroborate the comparison between the Pilates method and other types of exercises. In the other systematic review ${ }^{17}$, the authors included three studies that had not been published as articles in journals indexed in databases ${ }^{31,35,36}$. In their systematic review ${ }^{17}$, the authors evaluated the risk of bias according to the scale proposed by the Cochrane Back Review Group; however, they did not describe having performed a sensitivity analysis that considered the methodological quality of studies, although they did assess the risk of bias.

The results obtained in this review are related to effects that were analyzed after intervention because there are insufficient studies to conduct a meta-analysis comparing the effects of the Pilates method over time. Only one study ${ }^{30}$ has evaluated the Pilates method by comparing it with minimal intervention in the medium term, and it did not find a significant difference between the groups. One study $^{29}$ evaluated the Pilates method by comparing it to other types of exercises in the medium term, but it also failed to find a difference between the groups. The Pilates method has been used in rehabilitation programs, primarily to treat patients with chronic nonspecific low back pain. However, the effects of the Pilates method are only proven for patients with chronic nonspecific low back pain in the short term (immediately after intervention), and it is still not possible to make inferences regarding the effects of treatment over time. For this reason, more studies evaluating the effectiveness of this method for this population should be conducted. Furthermore, there are no studies that establish the standardized treatment duration, weekly frequency, intensity and type of exercises appropriate for patients with chronic nonspecific low back pain. The analyzed 
studies show some differences in terms of the choice of exercises because some used Pilates method-based floor exercises ${ }^{13,25-28,30}$ and others also used exercise equipment ${ }^{14,29}$. All of the studies opted to begin sessions with basic exercises, but the duration and frequency of sessions differed among the studies.

Studies of the Pilates method should better describe the goals of the exercises used in treatment. Few studies have explored the effect of springs and the variations in Pilates exercises that are methodologically different ${ }^{34,38}$. Perhaps for this reason, it is difficult to standardize an effective exercise program for rehabilitating specific groups of patients. Because there are few studies with a low risk of bias published on this topic, it is suggested that more studies on the effects of chronic nonspecific low back pain and that follow methodological quality criteria be undertaken and published.

Although it does not represent a direct limitation of this review, only a small number of studies were included because there are few indexed articles on the topic, and some studies were excluded because they offered comparisons that differed from those analyzed in the study. This small number of published articles reflected directly on the meta-analysis, as the analyses were performed using only a few articles. However, many studies are currently being conducted in different countries ${ }^{39,40}$, so this limitation should be overcome within a few years.

\section{- Conclusions}

The results of this systematic review suggest that Pilates method exercises are more effective than minimal intervention in improving pain and disability in the short term. However, they are not more effective than other types of exercise in reducing pain. Pilates method exercises can therefore be recommended for the improvement of pain and disability, but no definitive conclusion can be made regarding the analyzed outcomes (pain and disability) in the medium term. Further studies with a low risk of bias and larger samples should be published to obtain greater statistical power in the analyses.

\section{References}

1. Burton AK, Balague F, Cardon G, Eriksen HR, Henrotin Y, Lahad A, et al. Chapter 2. European guidelines for prevention in low back pain: November 2004. Eur
Spine J. 2006;15 Suppl 2:S136-68. PMid:16550446 PMCid:PMC3454541. http://dx.doi.org/10.1007/ s00586-006-1070-3

2. Van Tulder M, Becker A, Bekkering T, Breen A, Del Real MT, Hutchinson A, et al. Chapter 3. European guidelines for the management of acute nonspecific low back pain in primary care. Eur Spine J. 2006;15 Suppl 2:S169-91. PMid:16550447 PMCid:PMC3454540. http://dx.doi. org/10.1007/s00586-006-1071-2

3. La Touche R, Escalante K, Linares MT. Treating nonspecific chronic low back pain through the Pilates Method. J Bodyw Mov Ther. 2008;12(4):364-70. PMid:19083695. http://dx.doi.org/10.1016/j.jbmt.2007.11.004

4. Airaksinen O, Brox JI, Cedraschi C, Hildebrandt J, Klaber-Moffett J, Kovacs F, et al. Chapter 4. European guidelines for the management of chronic nonspecific low back pain. Eur Spine J. 2006;15 Suppl 2:S192-300. PMid:16550448 PMCid:PMC3454542. http://dx.doi. org/10.1007/s00586-006-1072-1

5. Walker BF. The prevalence of low back pain: a systematic review of the literature from 1966 to 1998. J Spinal Disord. 2000;13(3):205-17. PMid:10872758. http://dx.doi. org/10.1097/00002517-200006000-00003

6. Costa Lda C, Maher CG, McAuley JH, Hancock MJ, Herbert RD, Refshauge KM, et al. Prognosis for patients with chronic low back pain: inception cohort study. BMJ. 2009;339:b3829. PMid:19808766 PMCid:PMC2758336. http://dx.doi.org/10.1136/bmj.b3829

7. Waddell G. The Back Pain Revolution. 2nd ed. Edinburgh: Churchill Livingstone; 2004.

8. Menezes Costa Lda C, Maher CG, Hancock MJ, McAuley JH, Herbert RD, Costa LO. The prognosis of acute and persistent low-back pain: a meta-analysis. CMAJ. 2012;184(11):E613-24. PMid:22586331 PMCid:PMC3414626. http://dx.doi.org/10.1503/ cmaj.111271

9. Dagenais S, Caro J, Haldeman S. A systematic review of low back pain cost of illness studies in the United States and internationally. Spine J. 2008;8(1):8-20. PMid:18164449. http://dx.doi.org/10.1016/j.spinee.2007.10.005

10. Maher CG. Effective physical treatment for chronic low back pain. Orthop Clin North Am. 2004;35(1):57-64. http://dx.doi.org/10.1016/S0030-5898(03)00088-9

11. Latey P. Updating the principles of the Pilates method - Part 2. J Bodyw Mov Ther. 2002;6(2):94-101. http://dx.doi. org/10.1054/jbmt.2002.0289

12. Donzelli S, Di Domenica E, Cova AM, Galletti R, Giunta $\mathrm{N}$. Two different techniques in the rehabilitation treatment of low back pain: a randomized controlled trial. Eura Medicophys. 2006;42(3):205-10. PMid:17039216.

13. Gladwell V, Head S, Haggar M, Beneke R. Does a program of Pilates improve chronic non-specific low back pain? J Sport Rehabil. 2006;15(4):338-50.

14. Rydeard R, Leger A, Smith D. Pilates-based therapeutic exercise: effect on subjects with nonspecific 
chronic low back pain and functional disability: a randomized controlled trial. J Orthop Sports Phys Ther. 2006;36(7):472-84. PMid:16881464. http://dx.doi. org/10.2519/jospt.2006.2144

15. Posadzki P, Lizis P, Hagner-Derengowska M. Pilates for low back pain: a systematic review. Complement Ther Clin Pract. 2011;17(2):85-9. PMid:21457897. http://dx.doi. org/10.1016/j.ctcp.2010.09.005

16. Lim EC, Poh RL, Low AY, Wong WP. Effects of Pilatesbased exercises on pain and disability in individuals with persistent nonspecific low back pain: a systematic review with meta-analysis. J Orthop Sports Phys Ther. 2011;41(2):70-80. PMid:20972339. http://dx.doi. org/10.2519/jospt.2011.3393

17. Pereira LM, Obara K, Dias JM, Menacho MO, Guariglia DA, Schiavoni D, et al. Comparing the Pilates method with no exercise or lumbar stabilization for pain and functionality in patients with chronic low back pain: systematic review and meta-analysis. Clin Rehabil. 2012;26(1):10-20. PMid:21856719. http://dx.doi. org/10.1177/0269215511411113

18. Higgins JPT, Green S. Cochrane Handbook for Systematic Reviews of Interventions: Cochrane Book Series. Oxford: Cochrane Collaboration; 2008. http://dx.doi. org/10.1002/9780470712184

19. Elkins MR, Herbert RD, Moseley AM, Sherrington C, Maher C. Rating the quality of trials in systematic reviews of physical therapy interventions. Cardiopulm Phys Ther J. 2010;21(3):20-6. PMid:20957075 PMCid:PMC2941354.

20. Macedo LG, Elkins MR, Maher CG, Moseley AM, Herbert $\mathrm{RD}$, Sherrington C. There was evidence of convergent and construct validity of Physiotherapy Evidence Database quality scale for physiotherapy trials. J Clin Epidemiol. 2010;63(8):920-5. PMid:20171839. http://dx.doi. org/10.1016/j.jclinepi.2009.10.005

21. Moseley AM, Herbert R, Maher CG, Sherrington C, Elkins MR. PEDro scale can only rate what papers report. Aust J Physiother. 2008;54(4):288. http://dx.doi.org/10.1016/ S0004-9514(08)70017-5

22. Shiwa SR, Costa LOP, Costa LdCM, Moseley A, Hespanhol LC Jr, Venâncio R, et al. Reproducibility of the Portuguese version of the PEDro Scale. Cad Saúde Pública. 2011;27(10):2063-8. PMid:22031210. http:// dx.doi.org/10.1590/S0102-311X2011001000019

23. Maher CG, Sherrington C, Herbert RD, Moseley AM, Elkins M. Reliability of the PEDro scale for rating quality of randomized controlled trials. Phys Ther. 2003;83(8):713-21. PMid:12882612.

24. Borenstein M, Hedges LV, Higgins JPT, Rothstein HR. Introduction to Meta-Analysis. Chichester: John Wiley \& Sons; 2009.

25. Curnow D, Cobbin D, Wyndham J, Boris Choy ST. Altered motor control, posture and the Pilates method of exercise prescription. J Bodyw Mov Ther. 2009;13(1):104-11. PMid:19118799. http://dx.doi. org/10.1016/j.jbmt.2008.06.013
26. Da Fonseca JL, Magini M, De Freitas TH. Laboratory gait analysis in patients with low back pain before and after a pilates intervention. J Sport Rehabil. 2009;18(2):269-82. PMid:19561369.

27. Quinn K, Barry S, Barry L. Do patients with chronic low back pain benefit from attending Pilates classes after completing conventional physiotherapy treatment? Physiotherapy Ireland. 2011;32(1):5-12.

28. Rajpal N, Arora M, Chauhan V. The study on efficacy of Pilates and McKenzie exercises in postural low back pain -- a rehabilitative protocol. POTJ. 2008;1(1):33-56.

29. Wajswelner H, Metcalf B, Bennell K. Clinical Pilates versus general exercise for chronic low back pain: randomized trial. Med Sci Sports Exerc. 2012;44(7):1197205. PMid:22246216. http://dx.doi.org/10.1249/ MSS.0b013e318248f665

30. Miyamoto GC, Costa LO, Galvanin T, Cabral CM. Efficacy of the addition of modified pilates exercises to a minimal intervention in patients with chronic low back pain: a randomized controlled trial. Phys Ther. 2013;93(3):310-20. PMid:23064732. http://dx.doi. org/10.2522/ptj.20120190

31. O’Brien N, Hanlon M, Meldrum D. Randomised, controlled trial comparing physiotherapy and pilates in the treatment of ordinary low back pain. Phys Ther Rev. 2006;11:224-5.

32. Ostelo RW, Deyo RA, Stratford P, Waddell G, Croft P, Von Korff M, et al. Interpreting change scores for pain and functional status in low back pain: towards international consensus regarding minimal important change. Spine (Phila Pa 1976). 2008;33(1):90-4. PMid:18165753. http:// dx.doi.org/10.1097/BRS.0b013e31815e3a10

33. Macedo LG, Maher CG, Latimer J, McAuley JH. Motor control exercise for persistent, nonspecific low back pain: a systematic review. Phys Ther. 2009;89(1):9-25. PMid:19056854. http://dx.doi.org/10.2522/ptj.20080103

34. Hopewell S, McDonald S, Clarke M, Egger M. Grey literature in meta-analyses of randomized trials of health care interventions. Cochrane Database Syst Rev. 2007;(2):MR000010. PMid:17443631.

35. Anderson B. Randomized clinical trial comparing active versus passive approaches to the treatment of recurrent and chronic low back pain [thesis]. Miami, FL: University of Miami; 2005.

36. Gagnon L. Efficacy of Pilates exercises as therapeutic intervention in treating patients with low back pain [thesis]. Knoxville: University of Tennessee; 2005.

37. Quinn J. Influence of Pilates-Based mat exercise on chronic lower back pain [thesis]. Boca Raton, FL: Florida Atlantic University; 2005.

38. Queiroz BC, Cagliari MF, Amorim CF, Sacco IC. Muscle activation during four Pilates core stability exercises in quadruped position. Arch Phys Med Rehabil. 2010;91(1):86-92. PMid:20103401. http://dx.doi. org/10.1016/j.apmr.2009.09.016 
39. Da Luz MA Jr, Costa LO, Fuhro FF, Manzoni AC, De Oliveira NT, Cabral CM. Effectiveness of mat Pilates or equipment-based Pilates in patients with chronic non-specific low back pain: a protocol of a randomised controlled trial. BMC Musculoskelet Disord. 2013;14:16. PMid:23298183 PMCid:PMC3544561. http://dx.doi. org/10.1186/1471-2474-14-16

40. International Clinical Trials Registry Platform: Search Portal [Internet]. Geneva: World Health Organization [cited 2013 Mar 24]. Available from: http://apps.who.int/ trialsearch/default.aspx..

\section{Correspondence}

Cristina Maria Nunes Cabral

Rua Cesário Galeno, 445

CEP 03071-000, São Paulo, SP, Brasil

e-mail: cristina.cabral@unicid.edu.br 
Appendix 1. Search strategy.

\section{Search Strategy for MEDLINE (OVID)}

1. randomized controlled trial,pt.

2. controlled clinical trial.pt.

3. randomized.ab.

4. placebo.ab,ti.

5. drug therapy.fs.

6. randomly.ab,ti.

7. trial.ab,ti.

8. groups.ab,ti.

9. or/1-8

10. (animals not (humans and animals)).sh.

11.9 not 10

12. dorsalgia.ti,ab.

13. exp Back Pain/

14. backache.ti.ab.

15. exp Low Back Pain/

16. (lumbar adj pain).ti,ab.

17. coccyx.ti.ab.

18. coccydynia.ti,ab.

19. sciatica.ti,ab.

20. sciatica/

21. spondylosis.ti,ab.

22. lumbago.ti,ab.

23. or/12-22

24. pilates.mp

25. exercise therapy.mp

26. motor control exercise.mp

27. pilates based exercises.mp

28. exercise movement technics.mp

29. exercises movement techniques.mp

30. or/24-29

31. 11 and 23 and 30
Search Strategy for EMBASE (OVID)

1. clinical article/

2. exp clinical study/

3. clinical trial/

4. controlled study/

5. randomized controlled trial/

6. major clinical study/

7. double blind procedure/

8. multicenter study/

9. single blind procedure/

10. phase 3 clinical trial/

11. phase 4 clinical trial/

12. crossover procedure/

13. placebo/

14. or/1-13

15. allocate\$.mp.

16. assign\$.mp.

17. blind\$.mp.

18. (clinic\$ adj25 (study or trial)).mp.

19. compar\$.mp.

20. control\$.mp.

21. cross?over.mp.

22. factorial\$.mp.

23. follow?up.mp.

24. placebo\$.mp.

25. prospectiv\$.mp.

26. random\$.mp.

27. ((singl\$ or doubl\$ ou trebl\$ or tripl\$) adj25 (blind\$ or mask\$)).mp.

28. trial.mp.

29. (versus or vs).mp.

30. or/15-29 
31. 14 and 30

32. human/

33. nonhuman/

34. $\exp$ ANIMAL/

35. animal experiment/

36.33 or 34 or 35

37.32 not 36

38. 31 not 36

39.37 and 38

40.38 or 39

41. dorsalgia.mp.

42. back pain.mp.

43. exp LOW BACK PAIN/

44. $\operatorname{exp~BACKACHE/~}$

45. (lumbar adj pain).mp.

46. coccyx.mp.

47. coccydynia.mp.

48. sciatica.mp.

49. $\operatorname{exp~ISCHIALGIA/~}$

50. spondylosis.mp.

51. lumbago.mp.

52. or/41-50

53. pilates

54. exercise therapy

55. motor control exercises

56. pilates based exercises

57. exercise movement technics

58. exercise movement techniques

59. or $/ 53-58$

60. 40 and 52 and 59

Search Strategy for CINAHL (EBSCO)

S1. (MH “ Clinical Trials+”)

S2. "randomi?ed controlled trial*"

S3. "double-blind"

S4. "single-blind"
S5. "triple-blind"

S6. (MH "Placebo Effect")

S7. (MH "Placebos")

S8. "placebo*"

S9. "random*"

S10. (MH “Random Sample+”)

S11. (MH “Study Design+”)

S12. "latin square"

S13. (MH "Comparative Studies")

S14. (MH "Evaluation Research+”)

S15. (MH "Prospective Studies+")

S16. "follow-up stud*"

S17. "control*"

S18. prospectiv*

S19. "volunteer*"

$\mathrm{S} 20 . \mathrm{S} 19$ or $\mathrm{S} 18$ or $\mathrm{S} 17$ or $\mathrm{S} 16$ or $\mathrm{S} 15$ or $\mathrm{S} 14$ or $\mathrm{S} 13$ or $\mathrm{S} 12$ or $\mathrm{S} 11$ or $\mathrm{S} 10$ or $\mathrm{S} 9$ or $\mathrm{S} 8$ or $\mathrm{S} 7$ or $\mathrm{S} 6$ or $\mathrm{S} 5$ or $\mathrm{S} 4$ or $\mathrm{S} 3$ or $\mathrm{S} 2$ or $\mathrm{S} 1$

S21. (MH “Animals+”)

S22. S20 not S21

S23. Dorsalgia

S24. (MH "Back Pain+”)

S25. (MH “Low Back Pain”)

S26. "backache"

S27. "lumbar pain"

S28. (MH "Coccyx")

S29. (MH "Sciatica")

S30. "coccyx"

S31. "sciatica"

S32. Coccydynia

S33. (MH "Lumbar Vertebrae")

S34. (MH “Thoracic Vertebrae”)

S35. (MH "Spondylolisthesis")

S36. (MH "Spondylolysis")

S37. "lumbago" 
S38. S37 or $\mathrm{S} 36$ or $\mathrm{S} 35$ or $\mathrm{S} 34$ or $\mathrm{S} 33$ or $\mathrm{S} 32$ or $\mathrm{S} 31$ or $\mathrm{S} 30$ or $\mathrm{S} 29$ or $\mathrm{S} 28$ or $\mathrm{S} 27$ or $\mathrm{S} 26$ or $\mathrm{S} 25$ or $\mathrm{S} 24$ or $\mathrm{S} 23$

S39. pilates

S40. exercise therapy

S41. motor control exercise

S42. Pilates based exercises

S43. (MH “Therapeutic Exercise+”)

S44. S43 or S42 or S41 or S40 or S39

S45. S22 and S38 and S44

Search Strategy for CENTRAL - ONLINE (Cochrane Library)

1. MeSH descriptor Back explode all trees in MeSH products

2. MeSH descriptor Buttocks, this term only in MeSH products

3. MeSH descriptor Leg, this term only in MeSH products

4. MeSH descriptor Back Pain explode tree 1 in MeSH products
5. MeSH descriptor Back Injuries explode tree 1 in $\mathrm{MeSH}$ products

6. MeSH descriptor Low Back Pain, this tterm only in MeSH products

7. (low next back next pain) in All Fields, from 1800 to 2011 in all products

8. Ibp in All Fields, form 1800 to 2011 in all products

9. MeSH descriptor Sciatica, this term only in MeSH products

10. (\#1 or \#2 or \#3 or \#4 or \#5 or \#6 or \#7 or \#8 or \#9)

Search Strategy for PEDro

1. Low back pain

2. Pilates

3. Clinical trial

4. 1 and 2 and 3

Search Strategy for SciELO and LILACS

1. Pilates

2. Low back pain

3. 1 and 2 